\title{
Risk Perception and Knowledge in Fire Risk Reduction in a Dong Minority Rural Village in China: A Health-EDRM Education Intervention Study
}

\author{
Emily Ying Yang Chan ${ }^{1,2,3,4,5} \cdot$ Holly Ching Yu Lam ${ }^{1,3,5}$ - Phoebe Pui Wun Chung ${ }^{1,3}$ • \\ Zhe Huang ${ }^{1,3,5}$ - Tony Ka Chun Yung ${ }^{1,3,5}$ - Kelvin Wai Kit Ling ${ }^{3}$. \\ Gloria Kwong Wai Chan ${ }^{1,3,5} \cdot$ Cheuk Pong Chiu ${ }^{1,3,5}$
}

Published online: 17 September 2018

(C) The Author(s) 2018

\begin{abstract}
Fire is one of the major disasters in rural communities but evidence of the effectiveness of education interventions against fire risks is limited. This was a 2-year study assessed the effectiveness of face-to-face Health Emergency and Disaster Risk Management (HealthEDRM) education interventions for raising fire risk reduction knowledge in a fire-prone rural ethnic minority community. The study was conducted in various pre-set time points of an intervention-based project in a Dongbased community in Nanjiang Village, Guizhou Province in 2015 and 2016 to increase knowledge among the villagers about how to reduce general- and electrical-fire risks. Pre- and post-intervention questionnaires were used to evaluate the effectiveness of increasing fire risk-related knowledge through these interventions, immediately after the 2015 and 2016 interventions, and 17 months after the 2015 intervention. The knowledge of using fire blanket, recalling the correct emergency telephone number, unplugging unused electrical appliances, and not using
\end{abstract}

Emily Ying Yang Chan

emily.chan@cuhk.edu.hk

1 Collaborating Centre for Oxford University and CUHK for Disaster and Medical Humanitarian Response (CCOUC), The Chinese University of Hong Kong, Hong Kong, China

2 Nuffield Department of Medicine, University of Oxford, Oxford OX3 7BN, UK

3 The Jockey Club School of Public Health and Primary Care, The Chinese University of Hong Kong, Hong Kong, China

4 François-Xavier Bagnoud Center for Health and Human Rights, Harvard University, Boston, MA 02138, USA

5 Integrated Research on Disaster Risk (IRDR) International Centre of Excellence - CCOUC (ICoE-CCOUC), Hong Kong, China water to extinguish electrical fires had immediately improved after the interventions. Subjects demonstrated a better understanding that fire blankets can fight a blaze if used appropriately, and that knowledge was sustained for 17 months. The interventions were effective in improving fire prevention and response knowledge. Targeted interventions should be organized according to communities' culture, the evolution of economic prosperity and lifestyle practices.

Keywords Dong village · Fire risk education · Fire risk perception - Fire risk reduction - Health education $\cdot$ Rural China $\cdot$ Health-EDRM

\section{Introduction}

Fire is a severe global health hazard that causes great losses in disability-adjusted life years (DALYs) and damage (Peck 2011; Sim et al. 2017). Due to suboptimal fire extinguishing facilities and lack of timely disaster responses (Fu 2011; Tong 2011; Liao 2012, 2013; Wei 2013; Guo and $\mathrm{Wu} 2015$ ), rural communities that live in wooden housing structures and have lower socioeconomic status are usually at higher risk for fire disasters.

In southwestern China, fire disasters are common in the Dong ethnic minority tribe (Chen and Luan 2011; Fu 2011; Li 2014; Du and Okazaki 2016; Du et al. 2016b) because of traditional indoor fire pits for cooking, heat, lighting, and religious purposes, as well as the ever-increasing use of electrical appliances relating to, for example, heavily burdened sockets and aged electric wiring systems (Gan 2010; Tong 2011; Liao 2012; Wei 2013; Guo and Wu 2015; Du and Okazaki 2016; Du et al. 2016b). The Ganlan-style wooden houses that are built in close proximity without fire 
compartmentalization (a fire protection structure that contains room(s) for the purpose of limiting the spread of fire) in the mountainous areas also render the Dong community extremely vulnerable to widespread mountain fire incidents.

Health emergency and disaster risk management (Health-EDRM) (Chan and Murray 2017) refers to the systematic analysis and management of health risks surrounding emergencies and disasters. It plays an important role in reducing risks and vulnerabilities and expanding extensive preparedness, response, and recovery measures. Fire prevention knowledge and response training are examples of Health-EDRM for fire risk reduction. Despite existing fire protective measures, such as water storage tanks and mutual help groups, adopted within the Dong community (Xia and Tang 2009; Fu 2011; Liao 2013; Wei 2013; Du et al. 2016b), and law reforms with respect to buildings and infrastructures (Fu 2011; Tong 2011; Liao 2012; Yang and Wu 2012; Wei 2013; Guo and Wu 2015; $\mathrm{Xu}$ 2016), the fire accident rate in Dong communities remains high. More than 20 large-scale fires occurred in southern Dong ethnic communities in the past two decades (including Guizhou Province), and household fires remain the main disasters in Dong ethnic communities (Fu 2011; Liao 2012). Having a better understanding of risk perception and knowledge, as well as evaluating the effectiveness of, and knowledge gaps in, fire reduction will be useful for developing strategic fire risk reduction policies.

Previous studies have examined the effectiveness of improving building structures (Yang et al. 2006; Parbhoo et al. 2010; Jetten et al. 2011; Mashreky et al. 2011; Liu et al. 2012; Du and Okazaki 2016; Rybarczyk et al. 2016) and education sessions, particularly among children (Yang et al. 2006; Parbhoo et al. 2010; Jetten et al. 2011; Mashreky et al. 2011; Liu et al. 2012; Du and Okazaki 2016; Rybarczyk et al. 2016) in minimizing fire and burn risks. Several studies have also assessed the preparedness level and risk factors for fire accidents (Mashreky et al. 2008; Atiyeh et al. 2009; Mashreky et al. 2010; Peck 2011; Arshi et al. 2012; Scheven et al. 2012; Wei 2013; Zhou et al. 2014; Guo and Wu 2015; Du et al. 2016b; Gyedu et al. 2016; Rybarczyk et al. 2017; Smolle et al. 2017). However, there is a paucity of evidence for communitybased education interventions to enhance locals' knowledge and awareness of fire risks, especially in ethnic minority rural communities. The objectives of this study were to investigate the knowledge and fire risk perception and evaluate the effectiveness of a face-to-face community-based education intervention in increasing the awareness and knowledge of fire risks and related accidents in a fire-prone ethnic minority community in rural China. Findings from this study provide evidence to support the development of future community interventions that aim to increase preparedness for fire disasters among ethnic minority rural inhabitants.

\section{Methodology}

This is a 2-year serial cross-sectional survey study of a field-based health education intervention program that aimed to raise the knowledge level and awareness in fire prevention and response in a Dong ethnic community in Guizhou Province, China. Multiple field-based interventions were given in the form of drama and talks between 2015 and 2016. Intervention content was chosen based on the needs identified in the baseline assessment conducted in 2014 within the same community and was referenced to the guidelines issued by the Fire Department of the Ministry of Public Security of China. The effectiveness of the face-toface education intervention was evaluated by comparing the knowledge and awareness level to the topics in various time points of the study period using self-reported questionnaires.

\subsection{Study Site and Study Subjects: Nanjiang Village in Guizhou Province}

Fire disasters are common in Guizhou Province; 4221 fire cases were reported in the province in 2015 (SBOG 2015). In 2014, a 2-year project was established in Nanjiang Village, Guizhou Province as one of the Ethnic Minority Health Projects (EMHP) (Chan 2017). Its goal was to improve community health by delivering education programs in ethnic minority communities in rural China, especially for disaster-prone communities (Fig. 1). Nanjiang Village, a Dong-based community of about 1500 people, is located in the northwest of Shuikou Town in the southeastern part of Guizhou Province of southern China. The village consists of three sub-villages-Cenlan, Gao, and Cenwu. The village was recommended by the field project partner Wu Zhi Qiao Charitable Foundation to the project investigator. The study site was selected by the Ministry of Civil Affairs of the People's Republic of China for community development of the Wu Zhi Qiao Foundation from one of the 37 typical villages in Shuikou Town in Liping County, in the Qiandongnan Miao and Dong Autonomous Prefecture of Guizhou Province. The village relies on agriculture as the main source of income and remains one of the poorest villages in Shuikou Town, with an annual household income of RMB 1830 yuan, which is below the United Nations national poverty line $(<\mathrm{USD}$ 1.90/day per person) (World Bank 2015). In the following section, we outline the Health-EDRM intervention conducted in 2015 and 2016 to enhance disaster preparedness 


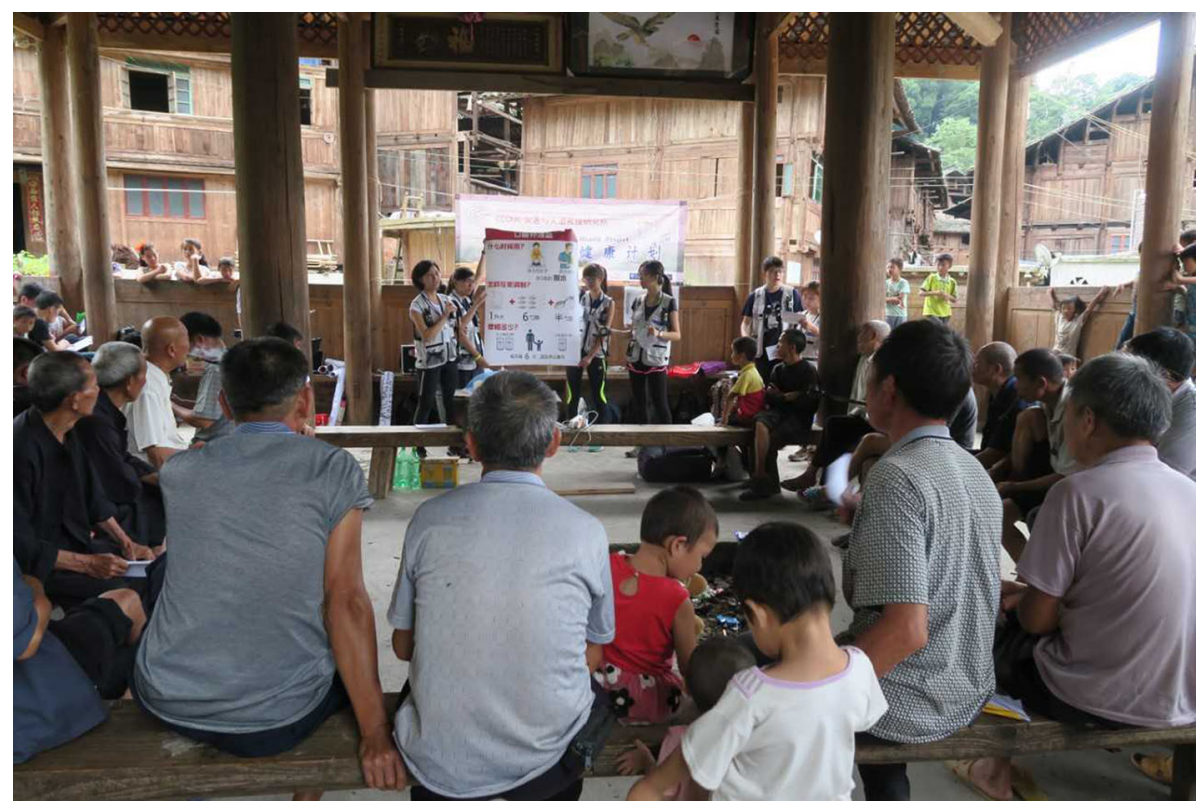

Fig. 1 Face-to-face Health-EDRM education intervention to increase fire risk reduction knowledge in Nanjiang Village, Guizhou Province, China. Photograph by Emily Y.Y. Chan, June 2016

literacy, in particular of fire risks among Nanjiang villagers.

\subsection{Field Health-EDRM Project Design}

This 2-year (November 2014-July 2016) face-to-face Health-EDRM education-based intervention focusing on raising the Dong ethnic minority community's knowledge, risk perception, and awareness of fire risk prevention and response in China was developed. The project consisted of an assessment of the community's health needs conducted in November 2014 and two tailored education interventions conducted in January 2015 and June/July 2016 based on the needs identified (CCOUC 2014, 2016). During the 2016 visit, an additional follow-up evaluation was conducted to test the retention of the intervention delivered in 2015. The outline of the data collection plan is presented in Fig. 2.

The 2014 health needs assessment included an in-depth interview with two village leaders, a focus group with 10 villagers, and an interviewer-administered survey with 66 respondents. The assessment indicated fire was a major disaster concern and the main identifiable causes of fire included improper use of electrical sockets and unattended indoor fires. These findings were consistent with the Guizhou Province fire report 2013-2016, which highlighted the major cause of fire accidents was related to electrical equipment (ranged from 42.3 to $49.7 \%$ ) (SBOG 2017). Due to villagers' requests and interests, fire riskrelated topics on general fire and electrical fire risk reduction were selected as the themes for the subsequent health education interventions. The content of these interventions were consistent with the Household Fire Safety Guideline issued by the national fire service (China Fire Service 2017) and was prepared to fit with the traditional culture and context of the village. For instance, guidelines related to fire accidents in high-rise buildings were not included in the interventions because the village buildings were no taller than three stories. Given the village's relatively low education level and economic status, fire blankets were introduced to the villagers instead of more expensive and complicated fire extinguishers. The education intervention on general fire was first conducted in 2015 and the electrical fire-related intervention was conducted in 2016 (Fig. 2).

Each of these intervention sessions took approximately 40 min to complete and was delivered in the form of dramas and interactive games. Banners and posters with illustrations were also used as visual aids to reinforce the messages (Chan and Wong 2017). During the intervention in 2015, villagers were taught to identify flammable materials such as washing powder, alcohol, matches, lighters, and plug sockets as well as the behaviors that can lead to fire accidents, burns, and electric shock. The correct method of using a fire extinguishing blanket and the evacuation strategy in case of major fires were also taught. During the 2016 intervention, two key messages, namely the prevention of fire and the response to fire accidents, were delivered. Content delivered included "not overloading electrical sockets with appliances"; "avoiding the placement of combustible items near electrical 


\begin{tabular}{|c|c|c|c|}
\hline \multicolumn{4}{|c|}{ November 2014 (Health needs assessment) } \\
\hline $\begin{array}{l}\text { Structured interview } \\
\text { - } 2 \text { village leaders (village } \\
\text { branch secretary, retired } \\
\text { doctor) } \\
\text { - Demographic features } \\
\text { of the village, livelihood } \\
\text { of villagers, health } \\
\text { status, disaster } \\
\text { experience and } \\
\text { preparedness, hygiene } \\
\text { and sanitation, waste } \\
\text { management }\end{array}$ & $\begin{array}{l}\text { Gender-speci } \\
\text { - } 10 \text { village } \\
\text { female: } 3) \\
\text { Health sta } \\
\text { health, ph } \\
\text { and menta } \\
\text { healthcare } \\
\text { disaster ex } \\
\text { preparedn }\end{array}$ & $\begin{array}{l}\text { ic focus group } \\
\text { s (male: } 7 \text {; } \\
\text { us (social } \\
\text { yical health, } \\
1 \text { health), } \\
\text { access, } \\
\text { perience and } \\
\text { ess }\end{array}$ & $\begin{array}{l}\text { Questionnaire survey } \\
\text { - } 66 \text { household } \\
\text { representatives } \\
\text { - Disaster risk perception, } \\
\text { disaster experience and } \\
\text { preparedness }\end{array}$ \\
\hline \multicolumn{4}{|c|}{ January 2015 (1st intervention) } \\
\hline \multicolumn{4}{|c|}{$\begin{array}{l}\text { Questionnaire survey (pre-post) } \\
\text { - } 64 \text { household representatives } \\
\text { - } \quad \text { Fire risk perception, fire disaster experience, fire protection and response measures }\end{array}$} \\
\hline \multicolumn{4}{|c|}{ June-July 2016 (2nd intervention) } \\
\hline \multicolumn{2}{|l|}{ Questionnaire survey } & \multicolumn{2}{|c|}{ Questionnaire survey (pre-post) } \\
\hline \multicolumn{2}{|c|}{$\begin{array}{l}\text { - } 101 \text { household representatives } \\
\text { Disaster risk perception, disaster } \\
\text { experience, fire protection and response } \\
\text { measures }\end{array}$} & \multicolumn{2}{|c|}{$\begin{array}{l}\text { - } 58 \text { household representatives } \\
\text { Electrical fire prevention and response } \\
\text { measures }\end{array}$} \\
\hline
\end{tabular}

Fig. 2 Outline of the 2014-2016 field program on fire risk and its data collection activities in Nanjiang Village, Guizhou Province, China

appliances"; and "unplugging electrical appliances when not in use to save energy" and prevent fire accidents. When experiencing fires that might have been caused by electric appliances/devices, villagers were reminded to "cut off the electricity supply immediately" and "not to use water to put out electrical fire." They were advised to escape to a safe and open area like the drum tower (their community focal point) and dial emergency number 119 to report the residential fire to prevent it from spreading and becoming a forest fire. A fire blanket and a disaster preparedness kit (including an emergency blanket, a torch, a flint, a whistle, a multipurpose knife, and an empty medication box) were distributed to the subjects who had completed both the preand post-intervention questionnaires as reminders of what they had been taught during the interventions. The program was conducted in Mandarin, and a summary of the key messages was given by a local translator.

\subsection{Study Subject Recruitment and Data Collection}

The samples in 2015 and 2016 were from the same sampling frame but were recruited independently. All subjects were aged 18 years or above and had provided oral consent. A combination of stratified sampling (by sub-village) and convenience sampling was applied for selecting participants in the 2014 needs assessment (except for the two village leaders), and the subjects in the 2015 intervention. Study subjects were recruited by using convenience sampling in the three sub-villages through home visits and leaflet distribution. One subject would be invited from each of the households of the community. Households with more than one family member who participated in the education activities would nominate a study qualified subject to join the study. During the 2016 visit, convenience sampling and snowball sampling were adopted for recruiting subjects in both the intervention and follow-up surveys without stratification. Again only one representative was invited from each household.

To evaluate the effectiveness of the interventions in raising the knowledge of fire risks among subjects, pre- and post-intervention interviewer-administered questionnaires were collected in both 2015 and 2016. The post-intervention questionnaires for the 2015 intervention were conducted twice: immediately after the intervention in 2015 and as a follow-up evaluation 17 months after the intervention in 2016. Of note, the original study design was to conduct the follow-up assessment 18 months after the intervention. Yet, due to logistical and weather issues, the second follow-up was conducted a month earlier than the original plan. For the second intervention in 2016, pre- and post-intervention (immediately after the intervention) questionnaires were collected. Knowledge enhancement 
Table 1 Questions in the evaluation surveys for the 2015 fire risk education intervention (pre-/immediately post-/17 months post-intervention) and the 2016 intervention (pre-/immediately post-intervention) in Nanjiang Village, Guizhou Province, China

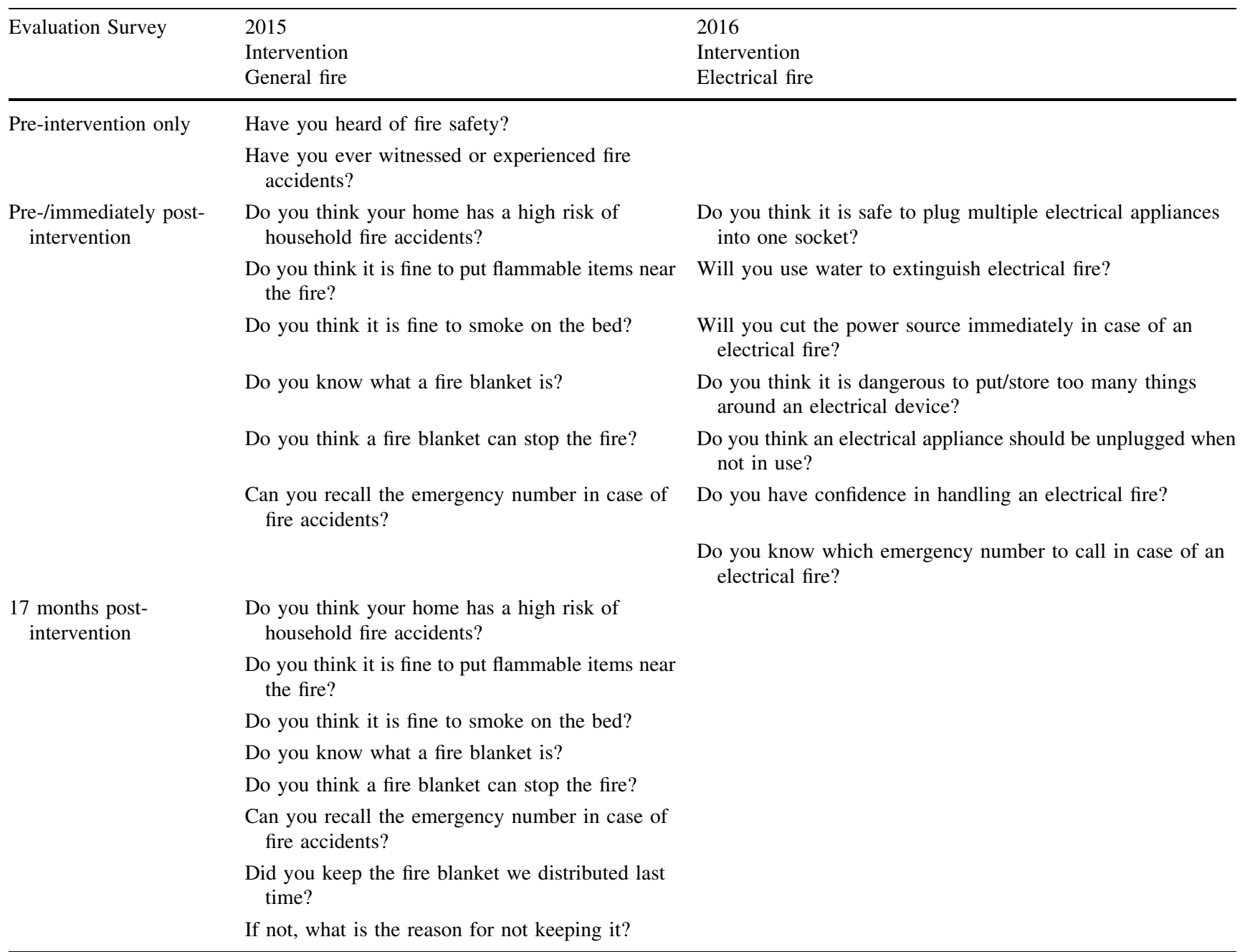

and retention on the topic of fire prevention and fire response, as well as the self-efficacy in responding to fire accidents were measured. The questions in the evaluation surveys are shown in Table 1.

\subsection{Statistical Analysis}

The data collected in Nanjiang Village were double entered and cleaned by trained researchers. Descriptive analysis of sociodemographic information of study subjects was generated and was compared with the general demographic information for Guizhou Province in 2010. McNemar's test was performed to compare the pre- and post-intervention questionnaires to assess the effectiveness of the interventions. During the revisit in 2016, simple logistic regression was used to evaluate the differences in knowledge and awareness between respondents who participated in the 2015 intervention and non-participants. The associations of demographic factors and experience of fire accidents with knowledge and awareness of fire risks at baseline were evaluated using multiple logistic regression. The significance level of this study was set at $p<0.05$. The data were analyzed using statistical software SPSS version 21.0. Ethics approval was obtained from the Survey and Behavioral Research Ethics Committee of The Chinese University of Hong Kong.

\section{Results}

A total of 64 household representatives participated in the general fire intervention in 2015. In 2016, 101 and 58 household representatives were interviewed for the 17-month follow-up survey and participated in the electrical fire intervention, respectively. The associated factors of fire risk perception, prevention and response awareness/knowledge at baseline were evaluated. 
Table 2 Sociodemographic characteristics of the households participating in the 2015 and 2016 fire risk education interventions in Nanjiang Village, Guizhou Province, China

\begin{tabular}{|c|c|c|c|}
\hline & $\begin{array}{l}\text { Nanjiang Village } 2015 \\
(n=57)^{\mathrm{a}}\end{array}$ & $\begin{array}{l}\text { Nanjiang Village } 2016 \\
(n=52)^{\mathrm{a}}\end{array}$ & Guizhou Province $2010^{\mathrm{b}}$ \\
\hline Male to female ratio & $1: 0.50$ & $1: 0.42$ & 1:1.069 \\
\hline Age (mean and SD) & $45.79 \pm 17.96$ & $48.54 \pm 16.62$ & NA \\
\hline \multicolumn{4}{|l|}{ Ethnicity, \% } \\
\hline Han & 1.75 & 0 & 64.30 \\
\hline Dong & 98.25 & 100 & 4.12 \\
\hline \multicolumn{4}{|l|}{ Occupation, $\%$} \\
\hline Farmer & 64.91 & 60.78 & NA \\
\hline Labor worker & 8.77 & 7.84 & NA \\
\hline Government official & 3.51 & 1.96 & NA \\
\hline Student & 1.75 & 1.96 & NA \\
\hline Housewife & 14.04 & 11.76 & NA \\
\hline Unemployed & 3.51 & 7.84 & 3.63 \\
\hline Retired & 1.75 & 5.88 & NA \\
\hline Others & 1.75 & 1.96 & NA \\
\hline \multicolumn{4}{|l|}{ Education level, \% } \\
\hline Illiterate & 25.00 & 21.15 & 8.74 \\
\hline No formal education & 5.36 & 7.69 & 9.52 \\
\hline Primary school & 37.50 & 44.23 & 39.37 \\
\hline Junior secondary & 21.43 & 25.00 & 29.79 \\
\hline Senior secondary & 8.93 & 1.92 & 7.28 \\
\hline Tertiary education & 1.79 & 0.00 & 5.29 \\
\hline
\end{tabular}

NA No available data, $S D$ standard deviation

a 64 (2015) and 58 (2016) household samples were collected but only 57 (2015) and 52 (2016) households completed the education intervention

b2010 Guizhou provincial data (SBOG 2015)

\subsection{The Sample Population}

During the study period, the total number of households was 286 (population size was 1442 people; average household size was 6.44 with standard deviation of 2.92 (CCOUC 2014)). A total of 64 Dong tribe household representatives $(22.4 \%)$ participated in the first intervention in 2015 while 58 household representatives (20.3\%) attended the second in 2016. For the 1-year follow-up for the 2015 intervention in 2016, 101 household representatives were interviewed for the follow-up evaluation survey, of which $29(28.7 \%)$ household representatives had participated in the 2015 intervention. Table 2 shows the sociodemographic characteristics of the subjects of the 2015 and 2016 interventions compared to Guizhou Province as a whole. The population characteristics in Nanjiang Village were quite different from those in Guizhou Province. The Nanjiang villagers were mainly Dong people and had a relatively lower education level.

\subsection{Associated Factors of Fire Risk Perception, Prevention and Response Awareness/Knowledge at Baseline}

Sociodemographic factors (age, gender, education level, and occupation) and previous experiences of fire disaster (self-reported direct and indirect fire accident experiences) were examined with logistic regressions to identify potential statistically significant associations among sociodemographic predictors and experiences with fire risk perception, knowledge of fire prevention, and responses to fire using the pre-intervention data in $2015(n=57)$. Significant results are presented in Table 3. Overall, as age group and education level were found to be statistically associated with fire risk perception and knowledge of emergency call number in univariate analysis, these variables were included in the final multiple logistic regression model with gender adjustment. The result of the logistic regression model indicated that younger age $(<45$ years) and higher education were associated with higher knowledge of recalling the emergency number. The odds ratio (OR) and 95\% confidence interval (CI) for knowing the 
Table 3 Sociodemographic factors that associated with fire risk perceptions and fire response awareness before the 2015 general fire hazards health education intervention in Nanjiang Village, Guizhou Province, China

\begin{tabular}{|c|c|c|c|c|}
\hline & \multicolumn{2}{|l|}{ Risk perception $(n=55)^{\mathrm{a}}$} & \multicolumn{2}{|c|}{ Recall emergency number for fire service $(n=55)^{\mathrm{a}}$} \\
\hline & $\begin{array}{l}\text { Simple logistic regression } \\
\text { OR }(95 \% \mathrm{CI})\end{array}$ & $\begin{array}{l}\text { Multiple logistic regression } \\
\text { OR }(95 \% \mathrm{CI})\end{array}$ & $\begin{array}{l}\text { Simple logistic regression } \\
\text { OR }(95 \% \mathrm{CI})\end{array}$ & $\begin{array}{l}\text { Multiple logistic regression } \\
\text { OR }(95 \% \text { CI })\end{array}$ \\
\hline \multicolumn{5}{|l|}{ Gender } \\
\hline Male & 1 & 1 & 1 & 1 \\
\hline Female & $0.43(0.08,2.20)$ & $0.16(0.02,1.11)$ & $0.69(0.23,2.04)$ & $0.20(0.03,1.21)$ \\
\hline \multicolumn{5}{|l|}{ Age } \\
\hline$<45$ & 1 & 1 & 1 & 1 \\
\hline $45-59$ & $0.13(0.02,0.71)^{*}$ & $0.10(0.02,0.72)^{*}$ & $0.24(0.07,0.83)^{*}$ & $0.18(0.03,1.08)$ \\
\hline$\geq 60$ & $0.00(0.00$, inf $)$ & $0.00(0.00$, inf $)$ & $0.13(0.03,0.61)^{*}$ & $0.04(0.01,0.36) *$ \\
\hline \multicolumn{5}{|l|}{ Education level } \\
\hline Primary or lower & 1 & 1 & 1 & 1 \\
\hline Secondary or higher & $4.62(1.13,18.93)^{*}$ & $2.36(0.43,12.97)$ & $6.13(1.92,19.54)^{*}$ & $4.41(1.07,18.18)^{*}$ \\
\hline \multicolumn{5}{|l|}{ Occupation } \\
\hline Farmer & 1 & - & 1 & - \\
\hline Not farmer & $2.00(0.51,7.81)$ & - & $1.97(0.69,5.64)$ & - \\
\hline
\end{tabular}

*Indicates $p$ value $<0.05$

${ }^{a} 64$ household samples were collected but only 55 samples were included in the regression due to missing data

emergency number for fire services (compared to those $<45$ years) for $45-59$ years and $\geq 60$ years were 0.18 (0.03-1.08) and $0.04(0.01-0.36)$, respectively. Respondents with an education at middle and higher levels were found to be 4.4 times more likely to recall the emergency number compared to those with a lower education level, the OR (CI) was 4.41 (1.07-18.18). Middle age (45-59 years) was associated with lower fire risk perception with an OR $0.10(0.02-0.72)$ (compared to those $<45$ years). Previous experience of fire accidents and other sociodemographic factors did not show obvious associations with risk perception and the studied knowledge.

\section{Evaluation of Health Education Intervention Effectiveness}

Fifty-seven (out of 64) and 52 (out of 58) sets valid preand immediately post-intervention questionnaires were collected for evaluation during the general fire education intervention (2015) and electrical fire education intervention (2016), respectively. The pre- and post-intervention questionnaires were compared in pairwise McNemar's test to evaluate the changes in knowledge level and awareness immediately after the interventions. In 2016, survey responses of 101 household representatives were included in the follow-up survey. Among these, 29 of them had participated in the general fire intervention in 2015. These 29 subjects were further evaluated for knowledge retention 17 months after the general fire intervention (2015) and their results were compared with those did not participate in the 2015 general fire intervention for fire knowledge differences.

\subsection{Intervention for General Fire Hazards in 2015: Immediately After Intervention}

Of the 64 households who participated in the 2015 intervention, $57(89.1 \%)$ valid pre- and post-intervention questionnaires were collected and analyzed. Seven questions about fire risk awareness, individual fire prevention awareness, and individual fire response awareness/knowledge were asked in the pre- and post-intervention surveys. Items with significant improvement are highlighted and the overall results are shown in Table 4.

The health education intervention was found to be statistically significant in enhancing individual fire response awareness and knowledge. The proportion of subjects who possessed knowledge of fire blankets increased from $12.5 \%$ $(n=7)$ to $52.6 \% \quad(n=30)$ after the intervention ( $p<0.005$, McNemar's test). Subjects who participated in the intervention were 1.5 times more likely to be aware of the use of a fire blanket to stop fire after the intervention (40\% before the intervention; $68.5 \%$ after the intervention; $p<0.005$, McNemar's test). Regarding knowledge of 


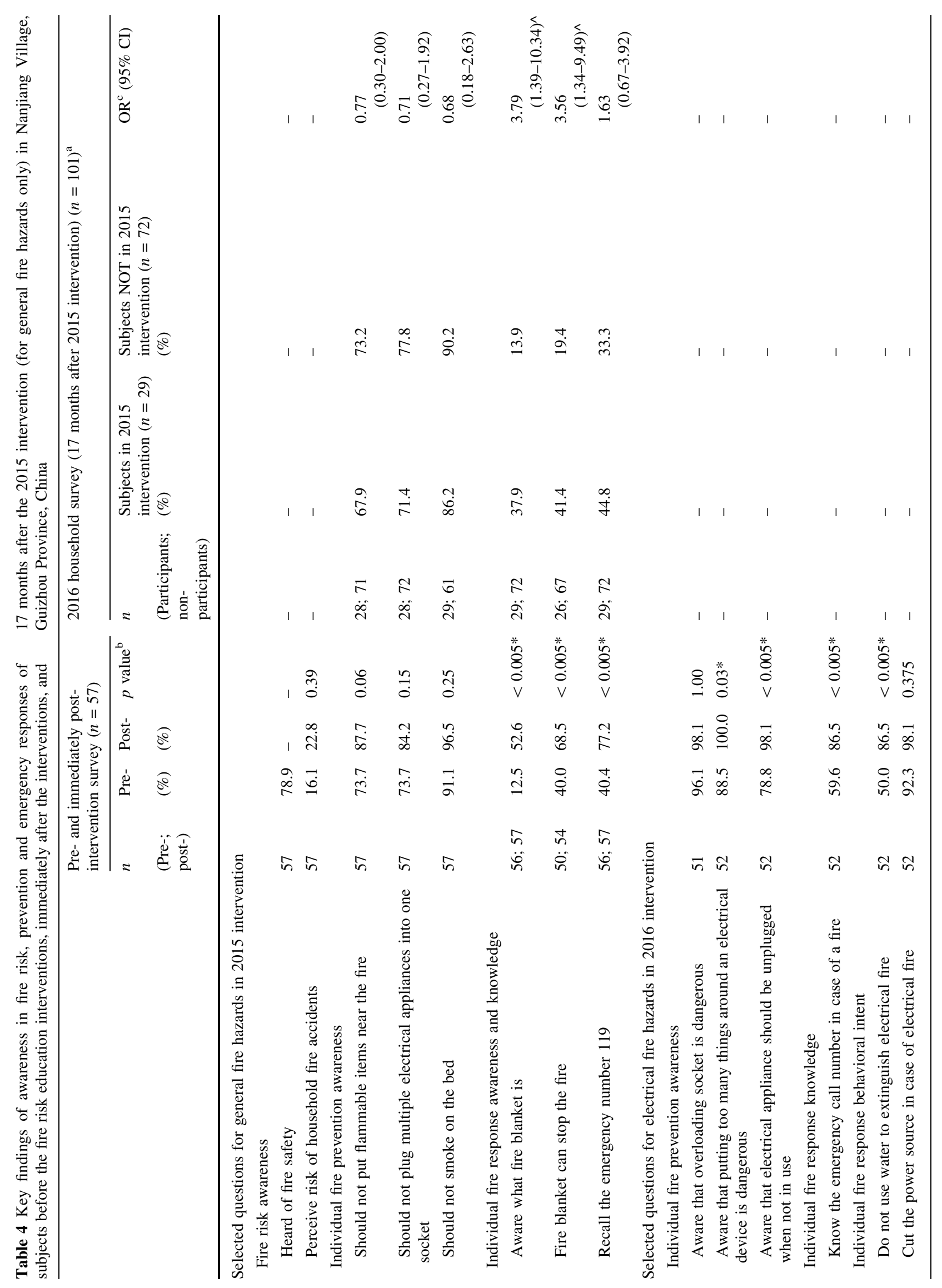




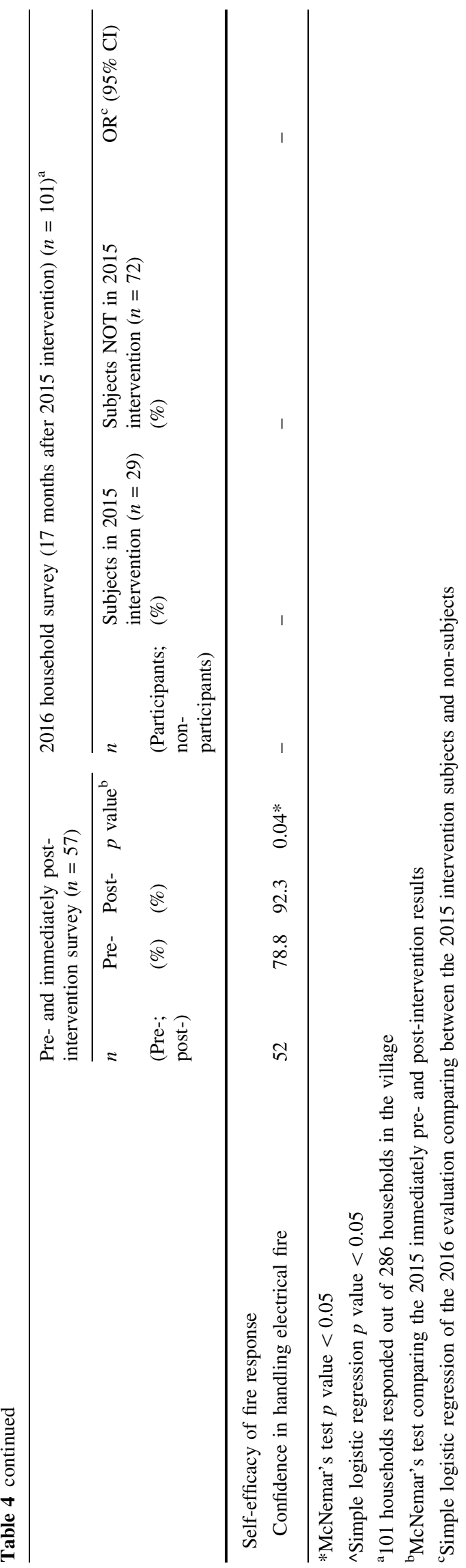

enhancement of fire response, $40.4 \%(n=23)$ of the subjects recalled the correct emergency number for fire services and $77.2 \%(n=44)$ possessed this knowledge after the intervention ( $p<0.005, \mathrm{McNemar}$ 's test). However, no significant improvement was observed for fire risk awareness and individual fire prevention awareness after the intervention (Table 4).

\subsection{Intervention for General Fire Hazards in 2015: 17 Months After Intervention}

Seven items including individual fire prevention awareness and individual fire response awareness and knowledge were tested (Table 4). The results show that the intervention was effective in enhancing subjects' knowledge on fire blankets after 17 months. Household representatives who had participated in the 2015 intervention demonstrated significantly better understanding on what a fire blanket is $(\mathrm{OR}=3.79,95 \% \mathrm{CI}=1.39-10.34)$ and understood that a fire blanket can fight a blaze if used appropriately (OR = 3.56, 95\% CI = 1.34-9.49). No significant differences were observed for other items (Table 4). Of note, among the 29 household representatives that participated in the 2015 health education intervention session, more than half $(n=19,65.5 \%)$ reported that they did not keep the fire blanket they received.

\subsection{Intervention for Electrical Fire Hazard in 2016: Immediately After Intervention}

Of the 58 households that participated in the intervention, $52(89.7 \%)$ valid pre- and post-intervention questionnaires were collected and included in the final analysis. Seven items for individual fire prevention awareness, individual fire response knowledge, individual fire response behavioral intent, and self-efficacy of fire response were tested in the pre- and post-survey (Table 4). Items with significant improvement are highlighted below and the overall results are shown in Table 4.

Regarding individual electrical fire prevention awareness, significant improvements were reported in the knowledge such as "putting many things around an electrical device is dangerous" and the need for "unplugging unused electrical appliances." Awareness was found to be increased from $88.5 \% \quad(n=46)$ to $100 \% \quad(n=52)$ ( $p=0.03$, McNemar's test) and $78.8 \%(n=41)$ to $98.1 \%$ ( $n=51)(p \leq 0.005$, McNemar's test), respectively. For individual electrical fire response knowledge, significant improvement was also found in the knowledge of "not using water to extinguish electrical fires" and the ability to recall the emergency number for fire services. Awareness scores were increased from $50.0 \%(n=26)$ to $86.5 \%$ $(n=45)(p \leq 0.005$, McNemar's test $)$ and $59.6 \%(n=31)$ 
to $86.5 \%$ ( $n=45)(p \leq 0.005$, McNemar's test), respectively. In terms of self-efficacy of fire response, the proportion of subjects who have confidence in handling electrical fires had increased from $78.8 \%(n=41)$ to $92.3 \%$ $(n=48)(p \leq 0.04$, McNemar's test $)$.

\section{Discussion}

This was the first study to examine the effectiveness of a face-to-face community-based health education intervention on risk perceptions, specifically the Health-EDRM knowledge of fire risks among high-risk ethnic minorities in rural China. The 17-month post-intervention evaluation showed that the awareness of the use of a fire blanket among people who had participated in the 2015 intervention was significantly higher (compared to non-participants in the 2015 intervention) while overall fire responses awareness among people who did not participate in the 2015 intervention remained low. However, fire risks perception continued to remain low in the community after the interventions. Previous experience of fire accidents did not show statistically significant association with risk perception and fire risks knowledge/awareness. Despite the high risk and repeated fire incidents in the study community, only a small proportion of respondents recalled the correct emergency number for fire services. This finding indicates low awareness of help seeking options from formal fire and relief agencies. Significant improvements were observed after the intervention in recalling the correct emergency number for fire services and in fire response awareness, especially in using a fire blanket and managing new hazards like electrical fires. Slight improvement in fire prevention awareness was also observed after intervention but the changes were not statistically significant. Villagers of younger age and higher education level were more likely to recall the accurate emergency number for fire services. These findings are consistent with other studies that knowledge and awareness level varies by age and education in both rural China and, for example, Ethiopia (Liao 2013; Wei 2013; Hajito et al. 2015).

In this study, study subjects generally possessed knowledge of general fire prevention. This may be explained by the prevalent official Dong village fire prevention education within the province that advises against smoking in bed or putting flammable firewood close to the fire pit and preventing children from playing with fire (Wei 2013). However, consistent with previous studies (Patil et al. 2010; Tong 2011; Wei 2013; Guo and Wu 2015), findings showed inadequate knowledge on fire response and new fire hazards (electricity safety), as well as low-risk perception among the rural population in general and the Dong ethnic people specifically. Similar results were reported in previous preparedness assessment and intervention studies that targeted ethnic minorities in rural China for fire prevention and responses (Wei 2013; Chan et al. 2014, 2017; Zhou et al. 2014; Guo and Wu 2015; Du et al. 2016a, b). Fire disaster response-related skill training such as fire risk inspection, firefighting training and drill (Tong 2011; Du et al. 2016b), emergency help-seeking capacity, and burn wound and injury management were limited.

Changes in fire risk awareness among rural community residents could be slow if health education and promotion are not available. Health education that focuses on familiarizing residents with firefighting facilities and emergency response may enhance locals' knowledge on health protection-related fire response behaviors (Stumpf et al. 2017). The evaluation of the tailored community-based health education intervention in this study shows the effectiveness in enhancing the knowledge and awareness in fire response and the prevention of new hazards, such as electrical fire, of the Dong minority community in the program. The effect of the intervention in promoting the knowledge of how to use a fire extinguishing blanket has sustained 17 months after the intervention. Contrarily, the awareness among those who did not attend the health intervention remained low and unchanged. Meanwhile, villagers' knowledge of electrical fire responses was inadequate. About half of the household representatives did not know water should not be used in putting out an electrical fire at baseline. As found in the focus group discussion during the 2014 visit, fire prevention education campaigns provided by the government tend to target mostly the prevention of general fire hazards rather than the prevention of new hazards (such as electrically caused fires) or related med$\mathrm{ical} / \mathrm{rescue}$ responses. With the increasing access and use of electricity in rural communities, electrical appliances have started replacing the traditional practice of using indoor active fire sources (for instance, for safety reasons, people would now prefer to use lamps for lighting and warming at night rather than a fire) (Wei 2013; Guo and Wu 2015). However, low awareness of electrical fire safety, particularly associated with unstable and overburdened low-capacity electricity systems (Wei 2013), has posed increasing electrical fire risks among the population in rural areas. Burn wounds caused by electrical burns might be internal and hidden, thus the severity of the injury may be underestimated, leading to delayed help-seeking behaviors. Electrical burns might also cause internal organ damage (for example, heart arrhythmia) and bleeding and may lead to life-threatening consequences (Pinto 2017; Runde 2018). Current general knowledge and implication of electrical burns on human health and its management in rural areas were insufficient. Health education should focus not only on prevention but also post-injury management to 
maximize impact of health education on and survival of the affected population (WHO 2018).

The low rate in recalling the emergency number for fire services among the subjects indicates that the awareness of formal help seeking options during fire accidents was low in the community. Studies in rural China showed that the locals mostly rely on community social capital and peer network such as neighbors and standard family more than the governments (Du et al. 2016b). Past research indicated that community-based interventions were the desired approach to deliver fire safety messages in rural areas because they take into account the culture specificity while forming a trusting bond in villages (Atiyeh et al. 2009; Du et al. 2016b). One previous study found that the national fire service emergency toolkit did not incorporate local information and many intervention programs did not take traditional cultures into account (Higgins et al. 2013). However, many risk factors are specific and inherent to the local customs and practices, like using a fire pit among the Dong population (Gan 2010; Tong 2011; Liao 2012; Wei 2013; Guo and Wu 2015; Du and Okazaki 2016; Du et al. 2016b). This study showed that community-based health intervention was effective in improving awareness of the emergency number for fire services. Thus, enhancing local knowledge and preparedness levels with culturally adapted interventions are crucially important.

Contrary to the association found among previous disaster experiences, such as the heightening of risk perception and preparedness intent in flood disaster (Chan et al. 2014; Gotham et al. 2018), experience with previous fire disasters did not seem to correlate with risk perception and preparedness intent. One possible explanation might be the community misconception that associates the decrease in fire hazards with their increasingly modern lifestyle (for instance the increasing trend of replacing indoor open fire sources with electrical appliances as well as the increasing general fire prevention awareness). Socioeconomic development and an urbanized lifestyle might bring along new fire hazard sources. Field program efforts to address fire risks should take note of the disassociation between disaster experiences and risk perception/preparedness behavioral intent. Future studies and education programs should re-examine how hazards, health risks, and behavioral intent might change with the evolution of economic prosperity and lifestyle practices.

A number of limitations exist in this study. First of all, randomized controlled trials would be a preferable design for intervention effectiveness studies. However, field program resources constraints would only allow an intervention study with pre-post evaluation study design but no control group for the evaluation attempts. Secondly, a single community study may limit the findings' generalizability. However, the study population of ethnic minority areas demonstrated sociodemographic characteristics that were different from the general population in Guizhou Province (Table 2). The study findings may be considered as a pilot investigation for Dong ethnic minority rural communities in developing Health-EDRM education interventions. Thirdly, fire risk awareness, knowledge level, and self-efficacy in this study were assessed using proxy questions. For instance, the knowledge of using fire blankets was used as a proxy for the knowledge in fire response. Together with the use of self-reported questions, reporting bias might be resulted. In addition, due to resources constraints during the 2016 visit, stratified sampling was not feasible and the final sample might be less representative in terms of residential location. Thus, there are limited possibility of comparing effects of the first intervention and the second intervention in a cohort manner. Barriers in culture and language would be other challenges but the support of experienced translators and community partners in this study has helped to minimize the limitations.

\section{Conclusion}

This study showed that risk perception, knowledge of new fire hazards and response were limited among this rural Dong ethnic minority community despite socioeconomic development. The tailored face-to-face community-based health education interventions were shown to be effective in increasing relevant awareness, knowledge level, and behavioral intent among subjects. Specifically, response knowledge regarding the use of fire blankets was significantly sustained after 17 months when comparing the program attendees with those who had not attended the interventions. However, contrary to previous studies, experience with previous fire disasters did not seem to correlate with risk perception and knowledge of fire prevention and response. In addition, as fire risk perception remained low after multiple interventions, fire prevention and response remains an important health and emergency disaster risk management effort. Targeted interventions should be needs oriented, specific to cultural norms, and organized for specific sociodemographic subgroups that have been found to have lower risk perception and knowledge levels. Further studies should aim to explore how hazards, health risks perception, and behavioral intent might change with the dynamics of community economic prosperity and lifestyle practices.

Acknowledgements This study was supported by the Jockey Club School of Public Health and Primary Care (SPHPC) Medical Humanitarian Response and Disaster Development Fund, Wu Zhi 
Qiao Charitable Foundation, Chow Tai Fook Charity Foundation, and The Hong Kong Jockey Club Charities Trust. The authors would like to express sincere appreciation to Sharon Chow, Carman Ka Man Mark, Sida Liu, Clare Lee, Edward Cheung, Dr. Xue Guo, Dr. Chunlan Guo, Prof. Benjamin Yip, Prof. Kevin KC Hung, Aman Yee, and Carol Wong, the field teams of CCOUC, the student participants of The Chinese University of Hong Kong, University of Hong Kong, and all the project volunteers for their valuable contributions to this intervention program.

Open Access This article is distributed under the terms of the Creative Commons Attribution 4.0 International License (http://crea tivecommons.org/licenses/by/4.0/), which permits unrestricted use, distribution, and reproduction in any medium, provided you give appropriate credit to the original author(s) and the source, provide a link to the Creative Commons license, and indicate if changes were made.

\section{References}

Arshi, S., H. Sadeghi-Bazargani, and R. Mohammadi. 2012. Burn injury-specific home safety assessment: A cross-sectional study in Iran. PloS One 7(11): Article e49412.

Atiyeh, B.S., M. Costagliola, and S.N. Hayek. 2009. Burn prevention mechanisms and outcomes: Pitfalls, failures and successes. Burns: Journal of the International Society for Burn Injuries 35(2): 181-193.

CCOUC (Collaborating Centre for Oxford University and CUHK for Disaster and Medical Humanitarian Response). 2014. The ethnic minority health project: Needs assessment trip report for Nanjiang, Guizhou. Hong Kong: CCOUC.

CCOUC (Collaborating Centre for Oxford University and CUHK for Disaster and Medical Humanitarian Response). 2016. The ethnic minority health project: Trip report for Nanjiang, Guizhou. Hong Kong: CCOUC.

Chan, E.Y.Y., and V. Murray. 2017. What are the health research needs for the Sendai Framework? The Lancet 390(10106): e35e36.

Chan, E.Y.Y., and C.S. Wong. 2016. Training manual on health and disaster preparedness in rural China. http://ccouc.org/_asset/file/ public-health-manual-eng-small.pdf. Accessed March 2018.

Chan, E.Y.Y., C. Guo, P.Y. Lee, S. Liu, and C.K.M. Mark. 2017. Health emergency and disaster risk management (HealthEDRM) in remote ethnic minority areas of rural China: The case of a flood-prone village in Sichuan. International Journal of Disaster Risk Science 8(2): 156-163.

Chan, E.Y.Y., J.H. Kim, C. Lin, E.Y.L. Cheung, and P.Y. Lee. 2014. Is previous disaster experience a good predictor for disaster preparedness in extreme poverty households in remote muslim minority based community in China? Journal of Immigrant and Minority Health 16(3): 466-472.

Chen, W., and C. Luan. 2011. Study on disaster prevention and mitigation in new countryside construction. Journal of Anhui Agricultural Science 39(7): 4099-4111 (in Chinese).

Du, F., and K. Okazaki. 2016. Building improvement responses to multi-hazard risk in the historic Dali Dong Village, Guizhou, China. International Journal of Disaster Risk Reduction 19(Supplement C): 64-74.

Du, F., H. Kobayashi, K. Okazaki, and C. Ochiai. 2016a. Research on the disaster coping capability of a historical village in a mountainous area of China: Case study in Shangli, Sichuan. Procedia - Social and Behavioral Sciences 218(Supplement C): $118-130$
Du, F., K. Okazaki, and C. Ochiai. 2016b. Disaster coping capacity of a fire-prone historical Dong Village in China: A case study in Dali Village, Guizhou. International Journal of Disaster Risk Reduction 21: 85-98.

Fire Department of the Ministry of Public Security. 2017. Guidelines for household fire safety. http://119.china.com.cn/txt/2017-09/ 19/content_40021390.html. Accessed March 2018 (in Chinese).

Fu, A.-H. 2011. Research on fire disasters and firefighting traditions in the Dong area of Qiandongnan. Journal of Original Ecological National Culture 3(2): 72-78 (in Chinese).

Gan, G. 2010. The firepit culture of the Dong people. Journal of Liuzhou Teachers College 25(3): 22-25 (in Chinese).

Gotham, K.F., R. Campanella, K. Lauve-Moon, and B. Powers. 2018. Hazard experience, geophysical vulnerability, and flood risk perceptions in a postdisaster city, the case of New Orleans. Risk Analysis 38(2): 345-356.

Guo, J., and D. Wu. 2015. The vulnerability of fire prevention systems in Dong villages: The case of the Dong villages in southeast Guizhou. Guangxi Ethnic Studies 5: 74-81 (in Chinese).

Gyedu, A., B. Stewart, C. Mock, E. Otupiri, E. Nakua, P. Donkor, and B.E. Ebel. 2016. Prevalence of preventable household risk factors for childhood burn injury in semi-urban Ghana: A population-based survey. Burns: Journal of the International Society for Burn Injuries 42(3): 633-638.

Hajito, K.W., H.A. Gesesew, N.B. Bayu, and Y.E.Tsehay. 2015. Community awareness and perception on hazards in southwest Ethiopia: A cross-sectional study. International Journal of Disaster Risk Reduction 13: 350-357.

Higgins, E., M. Taylor, M. Jones, and P.J.G. Lisboa. 2013. Understanding community fire risk-a spatial model for targeting fire prevention activities. Fire Safety Journal 62(Part A): 20-29.

Jetten, P., S. Chamania, and M. van Tulder. 2011. Evaluation of a community-based prevention program for domestic burns of young children in India. Burns: Journal of the International Society for Burn Injuries 37(1): 139-144.

Li, J. 2014. Analysis of fire disaster and fire safety management in rural areas. Fire Science and Technology 33(3): 343-345 (in Chinese).

Liao, J. 2012. The ecological anthropology thinking of fires of Dong nationality village and fire protection. Journal of Jishou University (Social Science Edition) 33(6): 110-116 (in Chinese).

Liao, J. 2013. On the indigenous prevention of fire disasters in Dong villages. Journal of Hunan University of Science \& Technology (Social Science Edition) 16(2): 38-41 (in Chinese).

Liu, Y, Y. Chen, J. Chen, X. Xu, and X. Liu. 2012. Characteristics of paediatric burns in Sichuan Province: Epidemiology and prevention. Burns: Journal of the International Society for Burn Injuries 38(1): 26-31.

Mashreky, S.R., A. Rahman, S.M. Chowdhury, S. Giashuddin, L. SvanstrOm, M. Linnan, S. Shafinaz, I.J. Uhaa, and F. Rahman. 2008. Epidemiology of childhood burn: Yield of largest community based injury survey in Bangladesh. Burns: Journal of the International Society for Burn Injuries 34(6): 856-862.

Mashreky, S.R., A. Rahman, T.F. Khan, L. Svanström, and F. Rahman. 2010. Determinants of childhood burns in rural Bangladesh: A nested case-control study. Health Policy 96(3): 226-230.

Mashreky, S.R., A. Rahman, L. Svanström, M.J. Linnan, S. Shafinaz, and F. Rahman. 2011. Experience from community based childhood burn prevention programme in Bangladesh: Implication for low resource setting. Burns: Journal of the International Society for Burn Injuries 37(5): 770-775.

Parbhoo, A., Q.A. Louw, and K. Grimmer-Somers. 2010. Burn prevention programs for children in developing countries require 
urgent attention: A targeted literature review. Burns: Journal of the International Society for Burn Injuries 36(2): 164-175.

Patil, S.B., N.A. Khare, S. Jaiswal, A. Jain, A. Chitranshi, and M. Math. 2010. Changing patterns in electrical burn injuries in a developing country: Should prevention programs focus on the rural population? Journal of Burn Care \& Research 31(6): 931-934.

Peck, M.D. 2011. Epidemiology of burns throughout the world. Part I: Distribution and risk factors. Burns: Journal of the International Society for Burn Injuries 37(7): 1087-1100.

Pinto, D.S. 2017. Environmental and weapon-related electrical injuries - UpToDate. UpToDate. 2 October 2017. https://www. uptodate.com/contents/environmental-and-weapon-related-elec trical-injuries?search=electrical\%20burn\&source=search result\&selectedTitle $=1 \sim 150 \&$ usage_type $=$ default\&display_ rank=1. Accessed March 2018.

Runde, D.P. 2018. Electrical injuries. https://www.msdmanuals.com/ professional/injuries-poisoning/electrical-and-lightning-injuries/ electrical-injuries. Accessed March 2018.

Rybarczyk, M.M., J.M. Schafer, C.M. Elm, S. Sarvepalli, P.A. Vaswani, K.S. Balhara, L.C. Carlson, and G.A. Jacquet. 2016. Prevention of burn injuries in low- and middle-income countries: A systematic review. Burns: Journal of the International Society for Burn Injuries 42(6): 1183-1192.

Rybarczyk, M.M., J.M. Schafer, C.M. Elm, S. Sarvepalli, P.A. Vaswani, K.S. Balhara, L.C. Carlson, and G.A. Jacquet. 2017. A systematic review of burn injuries in low- and middle-income countries: Epidemiology in the WHO-Defined African Region. African Journal of Emergency Medicine 7(1): 30-37.

SBOG (Statistical Bureau of Guizhou). 2015. Guizhou statistical yearbook 2015. http://www.gz.stats.gov.cn/tjsj_35719/sjcx 35720/gztjnj_40112/2015n/index_22.html. Accessed March 2018 (in Chinese).

SBOG (Statistical Bureau of Guizhou). 2017. Guizhou statistical yearbook 2017. http://www.gzstjj.gov.cn/tjsj_35719/sjcx_35720/ gztjnj_40112/2017/201712/t20171205_3077501.html. Accessed March 2018 (in Chinese).

Scheven, D., P. Barker, and J. Govindasamy. 2012. Burns in rural Kwa-Zulu Natal: Epidemiology and the need for community health education. Burns: Journal of the International Society for Burn Injuries 38(8): 1224-1230.

Sim, T., L. Dominelli, and J. Lau. 2017. A pathway to initiate bottomup community-based disaster risk reduction within a top-down system: The case of China. International Journal of Safety and Security Engineering 7(3): 283-293.

Smolle, C., J. Cambiaso-Daniel, A.A. Forbes, P. Wurzer, G. Hundeshagen, L.K. Branski, F. Huss, and L. Kamolz. 2017. Recent trends in burn epidemiology worldwide: A systematic review. Burns: Journal of the International Society for Burn Injuries 43(2): 249-257.

Stumpf, K., D. Knuth, D. Kietzmann, and S. Schmidt. 2017. Adoption of fire prevention measures-predictors in a representative German sample. Safety Science 94: 94-102.

Tong, H. 2011. Study on the countermeasures of Diaojiaolou fire protection in Guizhou. Journal of Guiyang College: Natural Science 6(3): 39-41 (in Chinese).

Wei, D. 2013. Establishment of a modern firefighting knowledge system in Dong ethnic villages. Journal of Guangxi University for Nationalities (Philosophy and Social Science Edition) 35(6): 103-107 (in Chinese).

WHO (World Health Organization). 2018. Injuries and disability: Priorities and management for populations affected by the earthquake and tsunami in Asia. http://www.who.int/violence_ injury_prevention/other_injury/tsunami/en/. Accessed $27 \mathrm{Feb}$ 2018.

World Bank. 2015. Global poverty line update. http://www.world bank.org/. Accessed 20 Aug 2018.

Xia, F., and W. Tang. 2009. An analysis of the Dong people's architecture and its cultural connotation. Journal of Guangxi Arts College (Arts Exploration) 23(2): 81-82 (in Chinese).

$\mathrm{Xu}, \mathrm{X} .2016$. Dong custom law on fire protection in the border area of Guizhou and Guangxi. Journal of Original Ecological National Culture 8(1): 76-81 (in Chinese).

Yang, J., C. Peek-Asa, V. Allareddy, C. Zwerling, and J. Lundell. 2006. Perceived risk of home fire and escape plans in rural households. American Journal of Preventive Medicine 30(1): $7-12$.

Yang, Z., and Q. Wu. 2012. The fire protection system of wooden buildings in Dong villages: A case study of Dong villages in Qiandongnan. Journal of Kaili University 30(5): 39-42 (in Chinese).

Zhou, B., X. Zhou, L. Ouyang, X. Huang, P. Zhang, M. Zhang, L. Ren, and P. Liang. 2014. An epidemiological analysis of paediatric burns in urban and rural areas in South Central China. Burns: Journal of the International Society for Burn Injuries 40(1): 150-156. 